\title{
L'expérience esthétique de l'environnement : une tension sociopolitique entre l'ordinaire et l'extraordinaire?
}

\author{
Anne-Sophie Devanne, Sophie Le Floch \\ Géographes, CEMAGREF, Avenue de Verdun, 33612 Cestas cedex, France
}

La question du lien entre esthétique et politique est au cœur de cet article. Les auteurs proposent, en inscrivant leur démarche dans les débats internationaux, un double dépassement de l'esthétique, celle du remarquable marquée par une vision élitaire allant jusqu'à masquer tout rapport au politique comme celle du quotidien qui tend à s'effacer comme telle au profit de l'utilitaire. L'enjeu est bien de rétablir la dimension politique qui est au cœur de tout rapport à l'esthétique. L'exploration d'un tel enjeu place nécessairement l'analyse au carrefour de plusieurs disciplines des sciences sociales.

La Rédaction

\section{Mots-clés :}

relations homme/ environnement : esthétique; enquête; Pyrénées; marais de la Sensée

\section{Keywords:}

human relationships with the environment; aesthetics ;

interviews ;

Pyrénées ;

Sensée marshes

Résumé - Concernant la dimension esthétique des relations des hommes à leur environnement, deux lectures dominent la littérature scientifique : d'un côté, une esthétique qui serait l'apanage d'un regard distancié, associée à l'idée d' " extraordinaire »; de l'autre côté, une esthétique qui, à force de puiser dans l'ordinaire, finirait par s'y dissoudre au profit d'autres valeurs dominantes utilitaires. À partir d'une analyse d'expériences de villégiature dans des contextes très différents, nous exposons que la dimension esthétique des relations des hommes à leur environnement peut être appréhendée comme une manifestation de la mise en tension entre ordinaire et extraordinaire. Une telle position permet de prendre toute la mesure de la dimension politique de l'esthétique, que l'expérience esthétique se fonde sur l'exclusion « d'autres » ou qu'elle se nourrisse « d'autres » et des liens sociaux qui les unissent.

\begin{abstract}
Viewing aesthetic experience of the environment as a socio-political tension between the ordinary and the extraordinary. When we consider aesthetics in human relationships with the physical environment as addressed in scientific literature, two leading approaches emerge. On the one hand aesthetics would appear to be the prerogative of a distanciated look and linked to the notion of "extraordinary". On the other, aesthetics would be so immersed in "ordinary life" that it finally dissolves in it and vanishes behind other leading values, such as utilitarian ones. This is particularly obvious in studies of local people's aesthetical experiences. In this paper, we argue that there could be a third way between these two approaches to understanding the aesthetical dimension of human relationships with the environment. We analyzed semi-directive interviews with people in two contrasted situations: mountain hikers in the Pyrénées and holiday-makers in the Sensée marshes of northern France. We point out that aesthetics in people's relationships with their physical environment may be seen as manifesting the tension between ordinary and extraordinary. This enables us to perceive the importance of the political dimension of aesthetics, whether visible (experience of a place explicitly incorporates the social relationships that take place in it) or concealed behind an aesthetical experience that appears to be very direct, material and autonomous.
\end{abstract}

Nul doute, l'esthétique intervient dans l'interprétation et dans l'appréciation que nous avons de ce qui nous entoure. La recherche de ce qui est beau, voire de ce qu'est la notion même de beau, concerne chacun dans sa vie quotidienne, qu'il s'agisse de se vêtir, de décorer son intérieur ou de choisir une destination de vacances.

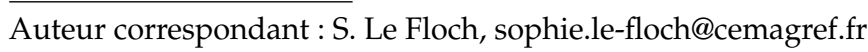

Nous nous intéressons ici à la dimension esthétique des relations des hommes à leur environnement, qui demeure relativement peu étudiée à ce jour. D'abord, nous rappellerons les principales orientations classiquement données à cette dimension dans la littérature scientifique existante. Ensuite, nous exposerons, puis illustrerons sur la base de travaux empiriques, nos objectifs et nos 
hypothèses, qui vont dans le sens d'une rediscussion des liens entre l'idée de beau et la notion d' " ordinaire » et, partant, des liens entre esthétique et politique - politique étant pris ici dans son sens générique, celui qui renvoie à tout ce qui a trait au collectif, aux rapports entre et au sein de groupes sociaux en vue de vivre ensemble.

\section{L'esthétique dans la littérature sur les relations des hommes à l'environnement}

Nous conduisons notre brève recension de la littérature relative à la dimension esthétique des relations des hommes à l'environnement en nous plaçant dans le champ des travaux qui adoptent un paradigme subjectiviste de l'esthétique. C'est-à-dire que nous retenons des travaux fondés sur l'idée que des éléments liés au sujet - culturels, sociaux, individuels - interviennent dans l'appréciation esthétique. Nous mettons donc de côté les approches objectivistes, qui postulent que la beauté d'un objet est inhérente à ses aspects formels ${ }^{1}$.

\section{Le paysage, production esthétique révélatrice de processus sociopolitiques}

La dimension esthétique des relations des hommes à l'environnement, considérée notamment au moyen de la notion de paysage, est essentiellement abordée depuis des questions d'ordre sociologique : il s'agit de voir en quoi la notion de paysage, en ce qu'elle rend compte d'une expérience visuelle, est révélatrice de processus sociaux (Rose, 2002). De nombreux auteurs s'intéressent, en effet, à la façon dont le paysage est mobilisé selon les époques et les catégories sociales, c'est-à-dire au paysage comme moyen de montrer - ostensiblement ou implicitement - l'appartenance à tel ou tel groupe social. Le paysage a ainsi pu être défini comme une manière de voir l'environnement socialement et culturellement déterminée, fondée sur l'adoption d'un regard distancié et contemplatif, et informée par des codes principalement façonnés par les artistes (Roger, 1978 ; Schama, 1995). Il serait le pays devenant progressivement, à partir de la Renaissance, spectacle à contempler pour des « regards exogènes » et cultivés (Donadieu, 1995 ; Corbin, 2001).

\footnotetext{
${ }^{1}$ Bien sûr, entre l'un et l'autre des deux pôles paradigmatiques, subjectiviste et objectiviste, des nuances dans les positions s'expriment. Il conviendrait sans doute d'examiner, par exemple, les apports d'une approche comme celle d'A. Carlson au propos développé ici : bien que rattaché au pôle objectiviste, son modèle d'esthétique cognitiviste souligne la nécessité d'adjoindre aux qualités formelles des objets des considérations sur ce qu'ils sont et sur la façon dont ils ont été produits. Cependant, puisque, de toute façon, nous ne visons pas l'exhaustivité, nous écartons cette approche, qui a été critiquée d'un point de vue humaniste (Dumas, 2001), celui que nous privilégions quant à nous - ainsi que nous l'exposons plus loin.
}

Mentionnons en particulier les apports de la géographie culturelle qui, en croisant la question de l'identité avec celle du pouvoir, insiste sur les processus de construction du paysage ; celui-ci, en tant que production esthétique, serait une matérialisation des luttes sociales, un moteur de la construction de la culture (Duncan et Duncan, 2001). Nombre d'auteurs explicitent la façon dont une élite montante, portée par le capitalisme naissant, aurait fait du paysage un instrument accompagnant et légitimant sa domination sociale et le contrôle qu'elle exerce sur le territoire (Cosgrove, 1985). Plus généralement, d'abondants travaux exposent que l'esthétique des objets et des lieux est évaluée surtout en termes d'adéquation entre des formes et des couleurs observées et les normes du «bon goût en vigueur (Baloglu et McCleary, 1999; Brush et al., 2000 ; Hunziker, 1995). Ou bien elle renvoie à l'impératif besoin de voir les lieux et monuments estampillés « remarquables » et de participer à une sorte de «collecte de regards » (J. Urry, cité par Harrison, 2001).

\section{De l'inféodation à l'« extraordinaire » à la dilution dans l'ordinaire}

En matière de rapports hommes/environnement, la dimension politique de l'esthétique est donc affirmée : toute production de paysage signifie l'appartenance à un groupe social. Cependant, si le contenu des paysages produits est fréquemment décrit par la littérature scientifique - les composantes de la pastorale, etc. -, la nature même de l'expérience paysagère a fait l'objet de relativement peu d'attention - la façon dont elle prend « corps » chez les personnes et les sens qui entrent en jeu (Rose, 2002). Une idée toutefois ressort : la production esthétique, en ce qu'elle crée de la distinction - sociale et spatiale simultanément -, consacrerait la rupture entre ordinaire et extraordinaire, et serait à rechercher du côté du second. Longtemps, dans la littérature scientifique consacrée aux paysages, ceuxci sont demeurés avant tout synonymes de "paysages remarquables ».

Des approches socioethnographiques ont été développées en réaction à ce que certains considèrent comme une posture élitiste - celle qui énonce que le paysage est l'apanage du regard de l'esthète et que les habitants d'un pays n'ont pas de paysage; ces approches sont un appel à revenir au «paysage des habitants ( $\left(\right.$ Sautter, 1979) ${ }^{2}$. Nous pouvons y voir des tentatives pour réconcilier esthétique et ordinaire dans les relations des hommes à l'environnement. Une lecture attentive révèle cependant que la dimension esthétique y est peu interrogée en elle-même ; elle disparaît peu ou prou derrière, voire dans, d'autres registres de valeurs.

\footnotetext{
2 Ce débat, particulièrement vif dans les années 1980-1990, anime la communauté scientifique française qui s'intéresse au paysage (Le Floch et al., 2004)
} 
En effet, lorsqu'ils visent à montrer que les habitants peuvent occasionnellement exprimer un sentiment esthétique à la vue de leur pays, la plupart des travaux décrivent le regard distancié et contemplatif qu'ils peuvent adopter, par exemple, à l'occasion d'un retour au pays après un voyage (de la Soudière, 1985) ou face à un observateur extérieur - comme un enquêteur (Cadiou et Luginbühl, 1995). Ce regard peut aussi être mobilisé au service d'une stratégie de promotion d'une activité locale. C'est ce que suggère J.-P. Deffontaines (1994) lorsqu'il propose de convaincre les agriculteurs que créer volontairement des formes « de qualité » revient à montrer à autrui «la modernité d'une agriculture vivante et intégrée dans des projets de société »; un champ aurait un " potentiel esthétique »- comme il a un potentiel agronomique -, qui ouvrirait à $l^{\prime}$ « intelligibilité » du lieu. Cette conception géoagronomique s'appuie sur le postulat que serait beau ce qui est intelligible, mais toujours au regard de l'observateur extérieur.

Certains travaux font ressortir l'idée que les "gens du pays » auraient bien une esthétique propre, au-delà de la conformation ponctuelle à un regard distancié. Mais, d'une part, leur idée du beau serait pratiquement insaisissable : il y aurait une réelle difficulté à exprimer ce qui relève des émotions personnelles, notamment dans le milieu agricole où ce registre est parfois opposé aux valeurs dominantes du travail et de la collectivité (de la Soudière, 1985). En outre, l'accès à l'« expérience privée ", à $l^{\prime}$ «acte intérieur » qu'est la perception serait loin d'être aisé et direct, même lorsqu'il s'agit de soi-même (Lenclud, 1995).

D'autre part, et surtout, l'esthétique ne nommerait $q^{\prime} u^{\prime} u n$ aspect des relations - dans ce qu'elles ont $d^{\prime}$ ' immatériel »-que les hommes nouent avec leur environnement quotidien; elle finirait par disparaître derrière ou dans d'autres registres dominants. Une idée courante est que le beau paysage est celui qui remplit de manière satisfaisante des fonctions utilitaires : produire pour nourrir les groupes humains ou bien être bien entretenu et accessible pour servir de support aux activités récréatives. Chez les agriculteurs en particulier, le « registre esthétique semble phagocyté par l'utilitaire», le mot «beau», détourné dans le sens des préoccupations dominantes (de la Soudière, 1985); ainsi, le beau champ est celui qui produit bien (Cudlinova et al., 1999; Van der Berg et al., 1998). En cela, ces auteurs rejoignent paradoxalement les tenants de la conception «élitaire» du paysage, pour lesquels le «beau paysage » de l'agriculteur n'est en fait que du «bon pays » (Cueco, 1995).

Il faut citer, tout de même, les travaux plus rares qui esquissent une esthétique de l'ordinaire. Par exemple, Y. Luginbühl (1989), dans ses efforts pionniers pour faire émerger l'idée de "paysage ordinaire ", décèle dans les paysages de la montagne bourguignonne les signes de reconnaissance de tout un groupe social qui perpétue les usages et les valeurs des petits vignerons, des ouvriers, des serfs, etc., et qui s'est construit en opposition aux «bourgeois » et aux «aristocrates » de la côte viticole. En outre, il met en évidence l'association des valeurs esthétiques et des valeurs de liberté, de poésie, d'imaginaire, en lien avec les pratiques sociales (Luginbühl, 1984).

Mais, pour l'essentiel, ce que les auteurs s'attachent à mettre en avant, $c^{\prime}$ est la façon dont toutes ces valeurs reflètent et nourrissent un sentiment d'appartenance à une communauté ou à un groupe social. Les auteurs insistent sur la dimension «symbolique» du paysage : pour eux, le paysage est symbole, c'est-à-dire qu'il représente, met une chose - abstraite - à la place d'une autre - concrète - (Voisenat et Notteghem, 1995). On peut aussi voir dans leurs travaux le sens fort et étymologique du terme de symbole, à savoir l'idée de la réunion de ce qui est disjoint. Le paysage serait porteur de signes de reconnaissance renvoyant à certaines identités collectives et formes de sociabilité locales, par distinction avec d'autres identités et d'autres formes de sociabilité ; la vue d'un paysage participant à la définition d'une identité sociale pourrait engendrer un certain plaisir esthétique. Pour le reste, l'existence de goûts propres aux habitants, plus qu'attestée et explorée, est postulée (Larrère, 1996), sur la base $\mathrm{d}^{\prime}$ « indices » recueillis dans les témoignages (de la Soudière, 1985).

\section{Une perspective humaniste phénoménologique}

Que trouvons-nous beau, de quoi est-il question à travers l'idée de beau, en quoi est-ce important de penser les relations à l'environnement en termes de beauté dans le contexte de nos actions quotidiennes? Nous tentons de proposer des éléments de réponse à ces questions en nous positionnant depuis un cadre théorique situé dans la tradition de la géographie humaniste phénoménologique.

\section{L'esthétique comme expérience humaine de l'environnement}

Cette tradition phénoménologique, bien qu'elle ne représente qu'une partie marginale de la discipline, joue un rôle déterminant dans l'affirmation de l'importance des "valeurs subjectives ", et en particulier de l'esthétique, dans les rapports des hommes à l'environnement. Sur un plan théorique, elle œuvre à délivrer l'esthétique d'une stricte inféodation à la vision et à l'art, à en faire un outil pour considérer l'indissociabilité du sujet et de l'objet et comprendre l'expérience humaine dans ses différentes dimensions sensorielles et sensibles. Elle se construit à partir des années 1960 et surtout 1970 en réaction à la nouvelle géographie, positiviste et quantitative, qui donne de l'homme 
une vision mécaniste et « désséchée ». D. Lowenthal, Y.-F Tuan, E. Relph et A. Buttimer définissent l'être humain comme un être social géographique, dont le rapport à l'étendue terrestre fonde significativement les conditions d'existence (Entrikin, 1976). Ils s'inscrivent dans la lignée d'E. Dardel (1990) qui redéfinit la notion de paysage, pertinente pour aborder « l'être-au-monde » de l'homme. En rupture avec la conception classique visuelle, le paysage devient déploiement, expression de l'existence de l'homme sur terre ; il exige un corps de chair, un regard incarné, une intentionnalité. Porteur de sens, il est insertion de l'homme dans le monde, base de son être social.

La perspective phénoménologique connaît actuellement un certain regain d'intérêt. Définissant $l^{\prime}$ «écoumène» comme «ce en quoi la terre est humaine, et terrestre l'humanité », le géographe A. Berque (2000) rejette l'idée d'une esthétique qui serait une affaire d'esthète imposant ses goûts, pour y voir plutôt ce qui touche tout un chacun dans sa vie de tous les jours. Dans le domaine de la philosophie, l'« engagement esthétique » d'A. Berleant (2007) puise également dans la tradition phénoménologique. Selon cet auteur, l'appréciation esthétique ne peut être réduite à un acte conscient de nature contemplative ; elle est inhérente à toute expérience - pas seulement artistique, mais plus largement environnementale - et implique la présence active du corps («embodied aesthetic »).

\section{Hypothèse : l'esthétique comme tension entre ordinaire et extraordinaire}

Ce cadre de pensée se prête à l'examen de l'hypothèse suivante : l'expérience esthétique n'émergerait pas exclusivement dans le regard distancié et culturellement informé dans un contexte de rupture avec l'ordinaire, mais dans la tension entre, d'un côté, l'ordinaire, le familier, le quotidien, et, de l'autre côté, l'extraordinaire, l'étranger, l'inhabituel. La notion de tension permet de considérer l'ordinaire et l'extraordinaire comme n'étant pas indépendants l'un de l'autre, ne se succédant pas dans le temps et dans l'espace, mais comme se construisant l'un par rapport à l'autre, se nourrissant sans cesse mutuellement.

Poser une telle hypothèse revient à privilégier un angle de questionnement peu exploré. En effet, il ne s'agit plus tant d'analyser la façon dont des groupes expriment leur existence, voire leur domination sociale ou culturelle, au travers de certaines productions esthétiques - paysages matériels ou représentations de paysages ; il s'agit plutôt d'examiner comment les personnes ordinaires font exister le paysage depuis leurs pratiques quotidiennes, en le rendant pertinent pour leurs propres vies. En d'autres termes, il s'agit de privilégier la question «comment le paysage se construit-il ? » par rapport à celle de " pour qui et pourquoi se construit-il ?» (Rose, 2002). C'est depuis un tel questionnement que nous proposons de réexaminer les liens entre esthétique et politique, dans le sens d'une réaffirmation de ceux-ci. Notre contribution à ce niveau porte sur deux points.

Le premier point concerne les exemples où l'esthétique est présentée comme ayant valeur en elle-même, indépendamment de toute considération sur les rapports aux autres - aux autres comme soi, aux autres différents de soi. J. et N. Duncan (2001) montrent que le paysage, en tant que processus de production esthétique, agit comme un mécanisme subtil mais hautement efficace d'exclusion, dans le sens où l'esthétique permet à la dimension politique d'avancer masquée. Celle-ci peut donc être démasquée : les relations sociales peuvent être identifiables et analysables non seulement à l'examen du processus de production esthétique, mais encore au travers des signes de leur absence dans les productions. Par exemple, R. Williams (1977) aborde la dissolution du paysan dans les représentations picturales de la campagne anglaise prisées par la bourgeoisie agrarienne du XVIII ${ }^{\mathrm{e}}$ siècle. Nous voudrions quant à nous illustrer le fait que, si les relations sociales peuvent figurer par leur absence, celle-ci est parfois explicitement énoncée par les groupes sociaux témoignant de leur expérience esthétique de l'environnement. L'analyse du contenu des productions esthétiques peut révéler l'éviction d' «autres » - autres que ceux qui regardent ou s'y représentent.

Le second point porte sur les exemples, beaucoup moins étudiés à notre connaissance, dans lesquels l'esthétique assume sa dimension sociopolitique, voire la revendique. La présence des autres - de certains autres et des liens qu'ils nouent est parfois vécue comme une dimension constitutive de l'expérience esthétique de l'environnement. En matière d'interprétation et d'appréciation de l'environnement, esthétique et politique seraient donc toujours liées, d'une façon ou d'une autre : en négatif - signes d'absence de liens sociaux, d'exclusion ; en positif signes d'existence de liens sociaux.

Pour développer nos hypothèses, nous nous basons sur l'analyse de récits d'expériences de personnes, recueillis dans deux situations de mobilité très différentes ${ }^{3}$. Nous nous inscrivons à la suite de travaux qui, tout en soulignant l'importance du beau et de l'expérience esthétique pour les personnes en situation de mobilité, ne présentent pas l'expérience touristique comme quelque chose en rupture avec le quotidien, mais comme un moment, un prolongement de ce quotidien (Harrison, 2001 ; Adler, 1989). Nous nous inspirons aussi de travaux récents

\footnotetext{
${ }^{3}$ Nous supposons que la tension ordinaire/extraordinaire, susceptible de s'exercer en maintes occasions de la vie quotidienne, serait à même d'être révélée plus particulièrement lorsque les personnes sont amenées à quitter momentanément leurs lieux de vie quotidiens pour leur plaisir. Nous pouvons classer les exemples rapportés ici principalement dans la catégorie «villégiature », un des types de mobilité d'agrément définis par J.-D. Urbain (2007), qui insiste sur l'importance de ce qui est vécu en termes d'installation dans un endroit donné.
} 
sur le paysage, qui présentent ce dernier non pas tant comme une image (culturelle) que comme un processus, comme le fruit d'une tension entre un premier plan - la vie ordinaire quotidienne - et un arrière-plan - la vie idéale imaginée - de la vie sociale ; les deux seraient les deux moments nécessaires pour nourrir une expérience du paysage (Hirsch, 1995).

D'une part, nous avons rencontré 24 marcheurs dans les Pyrénées centrales, partant dans des contextes variés et souvent plusieurs fois par an : vacances, week-end, résidence secondaire, sorties en club... (Devanne, 2005). Si beaucoup viennent de villes situées à proximité (Tarbes, Pau, Toulouse, etc.), certains n'hésitent pas à parcourir plusieurs centaines de kilomètres pour rallier leur lieu de villégiature. Des entretiens semi-directifs avec les marcheurs ont été conduits à des moments et dans des endroits différents (multiplication des contextes d'entretien). Nous les avons invités à parler, avec leurs propres mots, de leurs expériences de marche : de leur façon de les préparer, de leur ressenti sur place et de leurs souvenirs. D'autre part, nous avons repris un matériau constitué d'entretiens semi-directifs auprès de 26 visiteurs des marais de la Sensée (Le Floch et Eizner, 1997), limite naturelle entre les grands plateaux de l'Artois et la basse plaine de Flandre (Nord de la France). Depuis la fin du XIX ${ }^{\mathrm{e}}$ siècle, ces marais plantés de peupliers sont devenus un lieu attractif, synonyme de détente et de loisirs. De façon saisonnière, ils drainent toute une population, souvent modeste, venue des grandes villes du bassin minier et des environs, situées dans un rayon de 10 à $30 \mathrm{~km}$ : Arras, Douai, Lens, Valenciennes, Lille... Le temps d'un week-end ou de vacances, les innombrables caravanes, mobile homes et chalets qui parsèment les lieux s'animent, les berges se peuplent d'une foule de pêcheurs et de promeneurs.

\section{Des expériences esthétiques ordinaires}

\section{La mobilité : rupture paradoxale}

D'emblée, les personnes enquêtées présentent leur expérience de mobilité comme rupture avec l'expérience de la vie quotidienne; une rupture qui, en un paradoxe apparent, permet d'échapper au quotidien tout en y ramenant, est complément et opposé du quotidien. Il y a donc mise en tension entre deux moments qui contribuent, tous deux et par leurs apports mutuels, à l'existence des personnes.

Dans les Pyrénées comme dans les marais de la Sensée, la villégiature apparaît comme le lieu et le moment où l'on «se lave la tête » ou "se vide la tête », où l'on se «distrait »expressions fréquentes -, pour vivre autre chose et surtout pour mieux reprendre le train quotidien, " apaisé », "vidé de son agressivité », etc. Être là compte au moins autant que ce quel'on y fait et, si ceux qui fréquentent les Pyrénées se présentent comme des amoureux de la pratique de la marche, celle-ci apparaît parfois comme un prétexte ; parmi les enquêtés des marais de la Sensée, beaucoup revendiquent le droit de simplement s'asseoir et profiter du moment.

"Et puis c'est le fait d'être mieux au retour, hein! On le fait pour ça, se sentir bien. J'ai beaucoup marché quand je travaillais et que $j^{\prime}$ avais des choses difficiles à vivre, hein! Des tensions à vivre. [...] Et puis la semaine suivante, en retrouvant tout le monde, ben c'était bien! On avait un peu vidé son agressivité. » (Agricultrice, 60 ans, Pyrénées.)

"Ici, c'est l'évasion de la ville [...][qui permet de] se reposer de la maladie. On va revenir tout le mois de juillet; on sera reposé pour l'année, après! »(Femme sans profession, 60 ans, Sensée.)

Lorsque les enquêtés qualifient leur expérience de villégiature, c'est dans son opposition pied à pied à tout un ensemble d'aspects de la vie quotidienne : travail/détente, routine/découverte, difficultés relationnelles / renforcement des liens familiaux ou amicaux, maladie/convalescence, fatigue physique et nerveuse / remise en forme, etc. Ils suggèrent, dans leur façon de relater leur expérience, qu'on n'échappe jamais au quotidien, ne serait-ce que parce qu'il faut bien, à un moment, rentrer ; voire parce qu'il y a une tendance à projeter dans les lieux de villégiature les maux auxquels on était censé échapper en s'y rendant.

"Il y a trop de mobylettes! Je sais pas ce qu'ils font... À 3 sur une moto, des fois! Ils passent derrière, on a peur qu'ils viennent dans les mobile homes. On peut casser seulement par plaisir. [...] Ils recherchent sûrement la boisson. [...] Je ne vais pas dans le bois, je n'oserais pas. On attaque n'importe qui, vous savez, jeune ou vieux! (Femme sans profession, 60 ans, Sensée.)

La mise en tension de l'expérience quotidienne et de l'expérience de villégiature est donc une mise en tension de l'ordinaire et de ce qui sort de l'ordinaire - l'extraordinaire au sens littéral -, sans relever pour autant de l'exceptionnel - l'extraordinaire au sens fort. Les Pyrénées et les marais de la Sensée apparaissent alors comme des annexes de la ville pour leurs usagers respectifs, comme leur terrain de jeux, en quelque sorte.

\section{Le plaisir esthétique, jeu de regards entre réalité et rêve}

Cette mise en tension s'accompagne d'un plaisir, qui trouve une formulation explicite en termes esthétiques. Le plaisir esthétique de la contemplation visuelle, maintes fois rapporté lors des entretiens, est en particulier associé à l'idée de découverte de paysages nouveaux, intensément vécue : ce genre d'expérience "à vous couper le souffle »... Toutefois, l'expérience esthétique se loge aussi dans l'ordinaire, voire dans le plus familier. Ainsi, les objets les plus simples peuvent être dotés d'une dimension esthétique qu'ils n'auraient peut-être pas ailleurs, dans un autre contexte, et être source de plaisir autant que 
des choses moins courantes : les petites grenouilles ou les innombrables coccinelles, comme les marmottes ou les isards; les "églantiers avec leurs fruits rouges » ou les petites fleurs bleues des myosotis, comme les edelweiss ou les gentianes; les ruisseaux, avec « leur petit pont en bois ", comme les lacs d'altitude.

De même, les paysages les plus ordinaires peuvent être dotés d'une valeur toute particulière. Les enquêtés des marais de la Sensée précisent spontanément que les «beaux paysages » par excellence seraient à rechercher ailleurs : du côté de "la montagne», du « littoral dont les lumières inspirent les peintres ", voire des "marais de la Somme ». Et pourtant, leurs paysages à eux, c'est bien à ces lieux de villégiature qu'ils sont associés : ils sont beaux, mais pas tant en eux-mêmes, dans l'absolu, que dans ce qui les différencie et les rapproche à la fois de la ville et du quotidien; ils sont leur nature ordinaire, leur dépaysement dans le quotidien. «C'est beau ! S'il fait beau, c'est l'Amérique !» (Professeur, 48 ans.) Quant aux marcheurs en montagne, ils reconnaissent volontiers que les Pyrénées leur offrent la possibilité à la fois d'être presque "chez eux», dans « leur coin », et de se dépayser. Certes, les Pyrénées sont moins "grandioses» que les Alpes, moins « exotiques » que l'île de la Réunion, mais elles sont belles et cette beauté tient autant à leur familiarité qu'au plaisir de la découverte : «[Les Pyrénées,] ça me suffit. Je sais pas ce que les gens cherchent ailleurs, hein! » (comptable retraitée, 75 ans) ; "Tu fais 100 mètres, tu n'as jamais le même paysage devant toi » (assistante maternelle retraitée, 64 ans). Ainsi, même dans le connu, voire le familier, il est toujours possible de découvrir de l'inconnu, d'être surpris et d'en tirer un certain plaisir.

Il y a une sorte de jeu du regard, une manière d'exercer le regard qui engendre un plaisir esthétique, et qui est capable de l'engendrer de manière sans cesse renouvelée. Même dans le cas d'une marche autour d'un étang que l'on a faite maintes fois déjà dans sa vie :

"On a un étang, on peut faire le tour. On part par le chemin, on passe sous les arbres. Ça fait un tour superbe ", le regard traque les moindres mouvements, les moindres changements de l'environnement (secrétaire de mairie, 32 ans, Sensée). Pour certains marcheurs des Pyrénées, marcher en montagne, c'est forcément découvrir quelque chose, y compris lorsque l'on refait un parcours ou bien quand on fait un aller-retour: "Si tu es en montagne, un aller-retour, c'est pas gênant parce que tu n'as pas du tout les mêmes paysages... » (Institutrice à la retraite, 62 ans.)

Ce jeu du regard, qui traque ce qu'il pourrait doter de qualités esthétiques dans son environnement ordinaire, est passage vers le rêve - éventuellement en faisant intervenir d'autres sens, comme l'ouie ou l'odorat. L'enquête dans les marais de la Sensée révèle la récurrence et la force de la métaphore du vent dans les peupliers. Bougeant et bruissant sans cesse, ces arbres, qui n'ont pourtant rien de noble ni de mystérieux (Le Floch et Eizner, 1997), sont à la fois une prise sur la réalité et une invitation au rêve.
"Moi, ce que j'aime bien, c'est le bruit du vent. Quand il y a $d u$ vent, j'ouvre le cabanon derrière, je me mets dans le transat et j'écoute : j'ai l'impression d'entendre le bruit de la mer... » (Retraitée du commerce, 70 ans.)

Chez certains, notamment des marcheurs en montagne $^{4}$, la tension entre le monde de la réalité et le monde du rêve suscite une réflexion sur soi. Des personnes expriment leur plaisir immédiat d'avoir accompli une performance physique, de l'avoir emporté sur des éléments naturels a priori hostiles, d'avoir fait ce dont ils ne se savaient pas capables.

"Quand on est en haut d'un sommet un peu mythique, c'est vrai qu'on a une joie particulière. [?] On a l'impression, oh! tout modestement, mais enfin, d'avoir maîtrisé pas mal de forces de la nature, là. Sans illusion, mais enfin, on le vit comme ça quoi. (Rires)» (Cadre retraité, 72 ans.)

La relation esthétique à l'espace est donc présente, et même omniprésente, dans l'expérience de la villégiature conçue comme une mise en tension entre ordinaire et extraordinaire, entre premier plan - vie réelle - et arrièreplan-vie imaginaire-(Hirsch, 1995). Potentiellement, une telle mise en tension la fait surgir de tout lieu, à tout instant. Cependant, dans les témoignages, nous observons deux façons très différentes de présenter l'expérience esthétique, en particulier pour ce qui est des aspects renvoyant aux relations sociales, à une dimension collective.

\section{Esthétique et politique}

\section{Quand le paysage apprécié est ouvert et vide des autres}

Dans les récits de certains marcheurs dans les Pyrénées, l'idée de beau semble a priori autonome, fonctionnant seule et mettant en relation directe des personnes et un objet ou un environnement. La dimension politique de leur expérience n'apparaît pas d'emblée quand ils décrivent les paysages qu'il apprécient, et qui sont avant tout des paysages ouverts : point de vue depuis un sommet, paysage de crête, trouée dans la forêt, estives, lacs, etc. Pourtant, leur appréciation esthétique de ce qui les entoure n'est jamais exempte de considérations en termes de relations sociales, mais celles-ci apparaissent " en creux», dans le souhait de ne voir personne, dans l'éviction des autres.

"C'est certainement une des plus belles, enfin c'est ce que je trouve, une des plus belles [marches] du cirque, parce qu'elle est variée et? alors t'as? T'as la montée, ici, très raide, après t'as une immense plaine, là, que tu remontes. Ici c'est un coin magique aussi pour camper. On était tout seul. C'était magnifique. Après,

\footnotetext{
${ }^{4}$ Cette enquête a aussi révélé un jeu de regards entre imaginaire et réalité à travers l'usage que certains font de la carte, avant, pendant et après une marche (Devanne, 2005).
} 
la Table des Trois Rois, c'est un très, très beau sommet, avec une vue magnifique. Tu redescends dans un pierrier, tu fais glisser les pierres, c'est très rigolo. T'arrives à Mascaru, qui est aussi un endroit magique, et puis... tu redescends dans cette vallée d'Ansabère qui est très belle, par un endroit où y a personne, en plus, alors que tout le monde est là, quoi. Et en plus on était tout seul. » (Institutrice, 52 ans.)

En parlant de beau, ces marcheurs confèrent à leur expérience une dimension politique, où il est question $\mathrm{d}^{\prime}$ « autres » et de liens sociaux... ne serait-ce que du fait de leur absence. Les paysages ouverts, qui sont décrits comme fondateurs de l'expérience esthétique de la montagne, sont des paysages vides des autres. Si les marcheurs prennent un réel plaisir à «partager ce que l'on voit», en particulier lors de sorties avec leurs proches, le plaisir disparaît lors de rencontres jugées importunes. Une logique de distinction sociale est à l'œuvre, qui s'exprime en particulier dans le déni des «touristes », de la «masse » de ceux que l'on croise au départ des sentiers de randonnée et qui, « heureusement », ne poussent pas la randonnée trop loin, trop haut (Le Floch et Devanne, 2007).

"L'autre fois, on est allé camper avec mon oncle et ma tante, à un endroit où d'habitude y a personne, et justement y avait tout un... enfin, je sais pas si c'était un village ou une famille ou quelque chose comme ça, mais ils étaient tout un groupe de... peut-être une vingtaine [...] et j'avais pas du tout envie d'aller discuter au coin du feu avec eux. [...] En fait, ce que je recherche dans la montagne, ça m'énerve aussi un peu de le retrouver dans le regard des autres. »(Étudiant, 26 ans.)

Toutes les rencontres ne vont cependant pas à l'encontre de l'idée de plaisir. Certains ne cachent pas leur admiration lorsqu'ils aperçoivent un grimpeur suspendu à sa corde, le long d'une paroi. Certains, aussi, expriment tout le plaisir qu'ils ont à croiser un berger, à discuter avec celui qui, pour eux, représente le montagnard par excellence :

"J'aime beaucoup aller leur parler, comme ça, je vais trouver un berger... [...] C'est pas une curiosité; en fait, j'ai l'impression [...] qu'ils sont comme je vois les Pyrénées, quoi. Et j'ai envie de leur parler. » (Enseignant retraité, 66 ans.) Pour d'autres encore, il n'est pas désagréable de croiser d'autres randonneurs, mais en haut et loin du point de départ : ce sont des rencontres qui parfois rassurent (on n'est pas tout seul à être là) et qui souvent permettent de "voir qu'on n'est pas tout seul à faire d'énormes trucs, d'énormes efforts. Et que le plaisir est partagé » (institutrice retraitée, 62 ans).

Pour être en montagne, marcher en montagne et y prendre plaisir, c'est-à-dire en particulier faire l'expérience esthétique des paysages, il faut être en «bonne compagnie» et pouvoir contempler de grands paysages vides... de tous les autres. Une telle expérience esthétique n'est donc pas a-politique, elle est dé-politisée; plus précisément, sa dimension politique avance masquée (Duncan et Duncan,
2001) : c'est dans l'absence de l'autre qu'elle se construit. Les chemins de montagne apparaissent ici comme un espace ouvert - ils sont libres d'accès, souvent balisés -, mais pas pour tous - c'est du moins ce à quoi aspirent certains marcheurs. Un espace où, à l'opposé de l'espace public urbain, l'anonymat, la mixité des personnes et les rencontres fortuites ne sont pas de mise.

\section{Quand le paysage apprécié est animé et peuplé}

Il existe une autre façon d'apprécier le lieu de la villégiature qui, elle, se nourrit de la présence des hommes et de l'existence de liens entre eux; une présence qui ne se réduit pas seulement aux marques des activités humaines, mais qui peut aussi être une présence directe.

Une telle expérience caractérise les personnes en villégiature dans les marais de la Sensée et, dans une moindre mesure, certains marcheurs dans les Pyrénées. Pour eux, l'esthétique est liée au caractère animé et peuplé des lieux: l'animation découle autant des éléments - mouvement de l'eau, du vent - que de la vie animale - oiseaux, etc. - ou encore de la présence des hommes. Ces enquêtés décrivent des hommes - et de fait se décrivent - en mouvement, nouant des relations entre eux : soit par le regard - ils aiment se regarder les uns les autres -, soit par la parole -ils aiment échanger quelques mots lorsqu'ils se croisent-, soit par l'exercice d'activités communes - un pique-nique, un concours de pêche... L'idée de beauté, qui émerge de la tension entre l'ordinaire et l'extraordinaire, semble très proche de celle de la gaieté.

"L'été, c'est beau! L'hiver, c'est triste, il n'y a pas beaucoup de monde. Ce qui me plaît, c'est les gens qui se promènent : ils regardent les maisons. C'est gai, l'été. " (Femme sans profession, 52 ans, Sensée.)

"J'aime la nature, les oiseaux, les biches... J'aime regarder les pêcheurs... Surtout pendant les vacances : il y a des gens dans les caravanes qu'on peut regarder. Voir l'eau, les roseaux qui bougent, les canards... » (Mineur retraité, 52 ans, Sensée.)

Ce qu'ils aiment, c'est une scène familière où ils voient et décrivent les autres ainsi qu'eux-mêmes en mouvement. Un beau paysage est un paysage ouvert et plein des autres. Pas que des autres comme eux, c'està-dire des citadins en villégiature : les locaux - «les villageois »-y ont leur place aussi. Parmi nos enquêtés des marais de la Sensée, les citadins évoquent volontiers ceux qui habitent aux alentours immédiats des marais et qui y possèdent des terres ; ils savent bien, en particulier, que les peupliers qu'ils aiment tant et qu'ils qualifient parfois de "naturels », tant ils participent à cette image de nature qu'ils apprécient, sont plantés, entretenus et récoltés. Pour eux, c'est tout un ensemble de personnes qui façonnent le paysage des marais, en y projetant leurs goûts esthétiques, en le modelant aussi en fonction de ces 
goûts ${ }^{5}$ : les marais sont pleins de «gens qui regardent » et qui, ainsi, signifient ce qu'ils aiment regarder ; ils sont pleins de gens qui aiment qu'on les regarde ou qu'on regarde leurs petits chez eux, ces cabanons et ces jardinets aux décors créatifs et bigarrés ; les noms dont sont baptisés ces cabanons, à eux seuls, évoquent la revendication du droit d'être simplement là, d'apporter aux marais une touche personnelle...

Ce qui est créé, c'est une forme de nature socialisée, en prolongement de la vie quotidienne - en société et au foyer domestique -, dont elle ne propose finalement que des modalités de réalisation différentes. Ainsi, les activités et les formes de sociabilité permises par le plein air contribuent à renforcer les liens familiaux et, plus largement, sociaux, qui sont parfois malmenés dans le quotidien du travail et des obligations diverses. Les enquêtés évoquent le caractère rassurant, voire stimulant, d'un microcosme social où tout le monde se connaît et se fréquente sans toutefois qu'il y ait passage obligé par des formes de sociabilité plus «contraintes »: pas besoin de s'inviter, on se rencontre dehors, on improvise le partage d'un pique-nique ou une partie de pêche. Les lieux de villégiature ne sont pas seulement un cadre (symbolique, environnemental) où s'exercent les relations familiales, mais bien un aspect de ces relations, qui se présentent ici selon des modalités différentes par rapport à ce qu'elles sont en ville. Les formes spatiales, éphémères ou plus pérennes, qui naissent des relations nouées sont esthétiquement appréciées : un camping au bord de l'eau, un groupe qui pique-nique, un bivouac, un parking improvisé en terrain de pétanque.

L'expérience esthétique, ici, ne tente pas de masquer les relations sociales qui se déploient dans les lieux concernés : elle met au contraire en avant certaines de leurs formes et les valorise.

\section{Conclusion}

À partir d'une analyse de deux formes d'expériences de villégiature concernant des contextes - géographiques et sociaux - très différents, nous avons soutenu l'idée que la dimension esthétique des relations des hommes à l'environnement peut être appréhendée comme une manifestation de la mise en tension entre ordinaire et extraordinaire, entre vie réelle et vie rêvée. Une telle position permet de réaffirmer l'importance de la dimension politique de l'esthétique, que cette dimension avance masquée ou qu'elle soit revendiquée, que l'expérience esthétique se

\footnotetext{
${ }^{5}$ De cela, il ne faut pas déduire que les usagers des marais de la Sensée ne forment qu'un seul et même groupe social, homogène et consensuel : bien sûr, les villageois et les vacanciers, par exemple - voire les vacanciers entre eux - cherchent aussi, dans leurs témoignages, à se distinguer socialement les uns des autres sur certains points. Mais nous ne développons pas ici cet aspect.
}

fonde sur l'exclusion «d'autres » ou qu'elle se nourrisse « d'autres » et des liens sociaux qui les unissent.

Sur la base de ces résultats, il nous semble nécessaire de (ré)affirmer l'importance de l'esthétique dans l'expérience quotidienne que nous avons du monde qui nous entoure : loin d'être réservée aux regards "initiés », loin de ne surgir que dans des situations d'exception, elle émerge dans les mille petits dépaysements ordinaires que, tous, nous construisons au gré de nos mouvements. Cela devrait ouvrir la voie à une exploration de la multitude d'intensités et de contenus que l'appréciation esthétique est susceptible de revêtir. Cela devrait, en outre, conduire à s'interroger sur la juste distribution des droits à façonner l'environnement, ne serait-ce que par la simple expression de regards dotés de goûts propres et diversifiés; une interrogation qui amènerait à réfléchir aux conditions de discussion et de prise de décision collectives autour de la dimension esthétique des aménagements, afin que les personnes «ordinaires » puissent s'exprimer et être entendues (Mattila, 2002).

\section{Références}

Adler, J., 1989. Travel as performed art, American Journal of Sociology, 94, 6, 1366-1391.

Baloglu, S., McCleary, K.W., 1999. A model of destination image formation, Annals of Tourism Research, 26, 4, 868-897.

Berleant, A, 2007. Aesthetic Engagement and the Human Landscape. Communication au colloque international Environnement, Engagement esthétique et espace public : l'enjeu du paysage, Paris, LADYSS (CNRS), Université Paris X, Nanterre, 9 mai

Berque, A., 2000. Écoumène : introduction à l'étude des milieux humains, Paris, Belin.

Brush, R., Chenoweth, R.E., Barman, T., 2000. Group differences in the enjoyability of driving through rural landscapes, Landscape and Urban Planning, 47, 1-2, 39-45.

Cadiou, N., Luginbühl, Y., 1995. Modèles paysagers et représentations du paysage en Normandie-Maine, in Voisenat, C. (Ed.), Paysage au pluriel : pour une approche ethnologique des paysages, Paris, Éditions de la Maison des sciences de l'homme, 19-34.

Corbin, A., 2001. L'Homme dans le paysage, Paris, Textuel.

Cosgrove, D., 1985. Prospect, perspective and the evolution of the landscape idea, Trans. Inst. Brit. Geog., 10, 45-62.

Cudlinova, E., Lapka, M., Bartos, M., 1999. Problems of agriculture and landscape management as perceived by farmers of the Sumava Mountains (Czech Republic), Landscape and Urban Planning, 46, 71-82.

Cueco, H., 1995. Approches du concept de paysage, in Roger, A. (Ed.), La Théorie du paysage en France, 1974-1994, Seyssel, Champ Vallon, 168-181.

Dardel, E., 1990 [1 $1^{\text {re }}$ éd. 1952]. L'Homme et la terre : nature de la réalité géographique, Paris, CTHS.

Deffontaines, J.-P., 1994. L'agriculteur artisan producteur de formes, Natures Sciences Sociétés, 2, 4, 337-342.

Devanne, A.-S., 2005. Marcheurs en montagne et expérience de l'espace : une analyse de la construction du rapport à l'espace, à travers la pratique de la marche dans les Pyrénées. Thèse de doctorat en sciences de l'environnement, ENGREF, Paris. 
Donadieu, P., 1995. Pour une conservation inventive des paysages, in Roger, A. (Ed.), La Théorie du paysage en France, 1974-1994, Seyssel, Champ Vallon, 400-423.

Dumas, D., 2001. L'esthétique environnementale d'Allen Carlson. Cognitivisme et appréciation esthétique de la nature, Canadian Aesthetics Journal / Revue canadienne d'esthétique, 6 (http://www.uqtr.ca/AE/Vol $\_6 /$ index.html).

Duncan, J.S., Duncan, N., 2001. The aestheticization of the politics of landscape preservation, Annals of the Association of American Geographers, 91, 2, 387-409.

Entrikin, N., 1976. Contemporary humanism in geography, Annals of the Association of American Geographers, 66, 615-632.

Harrison, J., 2001. Thinking about Tourists, International Sociology, 16, 2, 159-172.

Hirsch, E., 1995. Introduction. Landscape: between place and space, in Hirsch, E., O'Hanlon, M. (Eds), The Anthropology of Landscape: Perspectives on Place and Space, Oxford, Oxford University Press, 1-30.

Hunziker, M., 1995. The spontaneous reafforestation in abandoned agricultural lands: perception and aesthetic assessment by locals and tourists, Landscape and Urban Planning, 31, 1-3, 399-410.

Larrère, R., 1996. Paysans, marché, paysages, C.R. Acad. Agric. Fr., 82, 4, 95-104.

Le Floch, S., Eizner, N., 1997. Le peuplier et l'eau, ou l'une des figures de la nature populaire, Le Courrier de l'environnement de l'INRA, 30, 19-28.

Le Floch, S., Deffontaines, J.-P., Terrasson, D., Ribéreau-Gayon, M.-D., 2004. Retour sur le «paradoxe du paysage » : dix ans de «paysage » dans Natures Sciences Sociétés. Séminaire de l'association NSS-Dialogues, Paris, 27 mai.

Le Floch, S., Devanne, A.-S., 2007. La «fermeture du paysage » : au-delà de l'esthétique, les enjeux d'un espace rural ouvert, in Berlan-Darqué, M., Luginbühl, Y., Terrasson, D. (Eds), Paysages : de la connaissance à l'action, Versailles, Quæ, 41-53.
Lenclud, G., 1995. L'ethnologie et le paysage. Questions sans réponses, in Voisenat, C. (Ed.), Paysage au pluriel : pour une approche ethnologique des paysages, Paris, Éditions de la Maison des sciences de l'homme, 2-17.

Luginbühl, Y., 1984. La montagne, un paysage de liberté pour le vignoble de Bourgogne, L'espace géographique, 1, 13-22.

Luginbühl, Y., 1989. Paysages élitaires et paysages ordinaires, Ethnologie française, XIX, 3, 227-236.

Mattila, H., 2002. Aesthetic justice and urban planning: who ought to have the right to design cities?, GeoJournal, 58, 131-138.

Roger, A., 1978. Nus et paysages : essai sur la fonction de l'art, Paris, Aubier.

Rose, M., 2002. Landscape and labyrinths, Geoforum, 33, 4, 455-467.

Sautter, G., 1979. Le paysage comme connivence, Hérodote, 16, 40-67.

Schama, S., 1995. Landscape and Memory, London, Harper Collins Publishers.

Soudière, M. de la, 1985. Le paysage à l'ombre des terroirs, Paysage et aménagement, 21-27.

Urbain, J.-D., 2007. Le touriste. Du sujet symptôme à l'homme qui rêve, Synergies pays riverains de la Baltique, 4, 15-25.

Van der Berg, A.E., Vlek, C.A.J., Coeterier, J.F., 1998. Group differences in the aesthetic evaluation of nature development plans: a multilevel approach, Journal of Environmental Psychology, 18, 141-157.

Voisenat, C., Notteghem, P., 1995. Avant-propos, in Voisenat, C. (Ed.), Paysage au pluriel : pour une approche ethnologique des paysages, Paris, Éditions de la Maison des sciences de l'homme, XI-XIII.

Williams, R., 1977. Plaisantes perspectives. Invention du paysage et abolition du paysan, Actes de la recherche en sciences sociales, 29-36.

Reçu le 20 mai 2007. Accepté le 6 mars 2008. 